\title{
An Energy-Efficient Sleep/Wake-up Routing Protocol for Wireless Sensor Networks
}

\author{
Dina El-Feky, Nada El-shennawy, Mahmoud Fahmy \\ Computer and Control Engineering Department, Faculty of Engineering, Tanta University, Tanta, Egypt \\ Email:engdina88@gmail.com
}

\begin{abstract}
In recent years, wireless sensor networks (WSNs) have a rapid development and they take a lot of research attention because of their wide-range applications. A WSN consists of a large number of distributed sensor nodes. These nodes are often deployed in remote or hostile areas to monitor physical or environmental conditions where they send this data to a main location. The most critical parameter in WSNs is network lifetime, so an efficient routing protocol is essential to reduce the energy consumption and to increase the network lifetime. This paper proposes an energy-efficient chain-based cooperative routing protocol based on node sleep/wake-up mechanism for WSNs. We compare this protocol with two efficient protocols; LEACH and CBCCP using MATLAB. Simulation results show that the proposed algorithm achieves better performance and conserves more energy than the other two protocols.
\end{abstract}

Keywords-Wireless sensor networks, Clustering, Energy consumption, sensing coverage.

\section{INTRODUCTION}

Wireless sensor networks (WSNs) support a wide range of applications, such as manufacturing, health needs, home automation, smart building and monitoring disaster areas [1]. In a large-scale WSN, high-density sensor nodes are used. These sensor nodes are equipped with limited batteries, and it is difficult to replace or recharge these batteries, so efficient routing protocols should be designed to reduce energy consumption and to prolong the network lifetime. Scheduling the node is also of prime importance to increase the network lifetime by turning off redundant nodes and activating another set of nodes, providing good sensing coverage.

WSN routing protocols can be classified as flat, hierarchical and location-based [1]. In a flat structure, all nodes do the same role. The base station transmits requests for different areas and waits for the sensor response, such as Sensor Protocols for Information via Negotiation (SPIN) and Direct Diffusion (DD) [2]. In hierarchical routing protocols, sensor nodes have different roles; this type also called cluster routing. Cluster routing processes have two steps: cluster selection and routing as in Low-Energy Adaptive Clustering Hierarchy (LEACH) [3]. In location-based, the node position is the main parameter in this routing as in Geographical Adaptive Fidelity (GAF) [4].

Power-Efficient Gathering in Sensor Information Systems (PEGASIS) [5] decreases energy consumption and increases the lifetime of the network by communicating nodes only with the closest neighbors to them. One of the nodes on the chain acts as a head node. The head node is responsible for forwarding data to the main station. The performance of
PEGASIS protocol is better for twice at least than LEACH protocol.

In Energy-LEACH (E-LEACH) protocol [6], cluster head is selected by taking into account the residual energy of each node. In the first round, a cluster head $(\mathrm{CH})$ is selected randomly as the same in LEACH protocol. In the next rounds, the cluster head is selected based on the residual energy of each node. This means that the node, which has more residual energy, is selected as the $\mathrm{CH}$.

In Node Ranked LEACH (NR-LEACH) protocol [7], the cluster head is selected based on the residual energy of each node, the number of connections with other nodes and the distance of the node from the base station. The result shows that NR-LEACH increases network lifetime comparing with other LEACH protocol.

The Chain Based Cluster Cooperative Protocol (CBCCP) [8] uses two kinds of communication at intra and inter cluster level to add extra advantages. Reducing energy in communication to increase the lifetime of the network is the main goal of CBCCP. The results show that CBCCP outperforms LEACH because CBCCP depletes less energy.

In this paper, an energy efficient routing protocol is proposed based on the node energy to increase the total network lifetime by using a sleep/wake-up mechanism. The proposed algorithm is implemented and evaluated using MATLAB. It is compared with LEACH [3] and CBCCP [8]. The paper is organized as follows. Section II introduces the architecture of wireless sensor networks. In Section III, routing and scheduling protocols are reviewed. In Section IV, the proposed protocol is presented. The performance comparison of the proposed protocol with LEACH and CBCCP is shown in Section V. In Section VI, conclusions and trends for future work are given.

\section{W IRELESS SENSOR NETWORKS (WSNS)}

WSNs are one of the most developing technologies. A WSN consists of spatially distributed sensing nodes, which are used to control environmental or physical conditions, as pressure, sound, motion, temperature, vibration or pollutants, and send this data to the base station [1] as shown in Fig. 1. The sensor node is the main component in a WSN. Each node has four basic units: sensing unit, processing unit, transceiver unit and power unit, as shown in Fig. 2. There are many additional components such as a power generator, mobilizer and location finding system. 


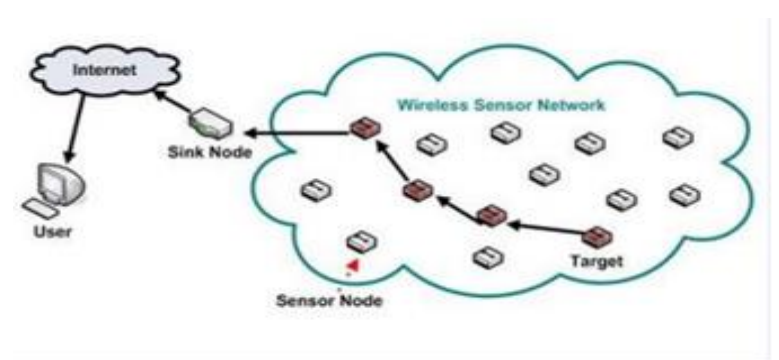

Figure 1. Wireless sensor network architecture.

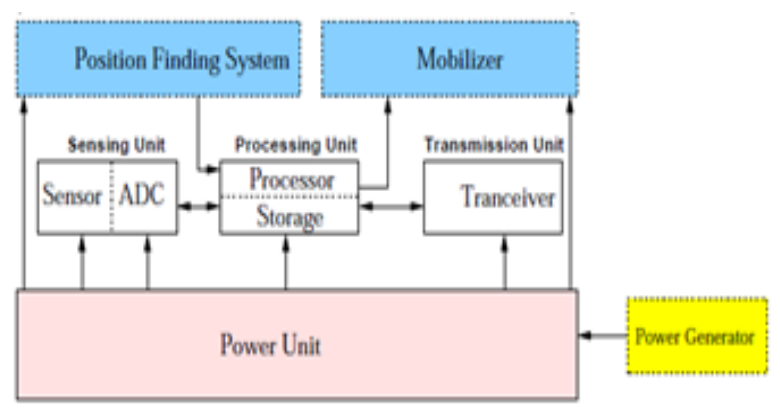

Figure 2. Components of sensor node.

The sensing unit contains two parts: sensors and analogto-digital converters. The sensors generate analog signals and the analog-to-digital converters convert them to digital signals. The processing unit is responsible for managing the procedures to make the cooperation between sensor nodes to execute the specified sensing tasks. The transceiver unit is responsible for connecting sensor nodes to the network [1].

\section{ROUTING PROTOCOLS}

In a WSN, sensor nodes communicate with each other to transmit collected data to the main location. This is the major role of routing algorithms. Sensor nodes are equipped with a low battery and it is hard to replace or recharge these batteries, so WSN lifetime is limited. To prolong WSN lifetime, we need efficient routing and scheduling algorithms. There are many routing protocols that are used with WSN such as LEACH [3] and CBCCP [8].

\section{A. LEACH Protocol}

LEACH [3] was proposed to decrease energy consumption and to increase the network lifetime. The operation of LEACH is divided into rounds as shown in Fig. 3. Each round has two steps. The first is a setup step where nodes are divided into clusters, whereas the second step is a steadystate phase where data is collected and sent to the cluster head $(\mathrm{CH})$ and then to the base station.

LEACH uses the received signal strength of nodes to organize clusters. Depending on the probability function, each node decides to be a $\mathrm{CH}$ or not. Then, the $\mathrm{CH}$ nodes send advertisement messages to the other nodes with the same transmit energy. The other nodes choose their cluster based on the $\mathrm{CH}$, which will be connected with the minimum energy that is used to pass data to $\mathrm{CH}$. Finally, the cluster is organized and nodes can send data to $\mathrm{CH}$ that collects data and passes it to the main location [3].

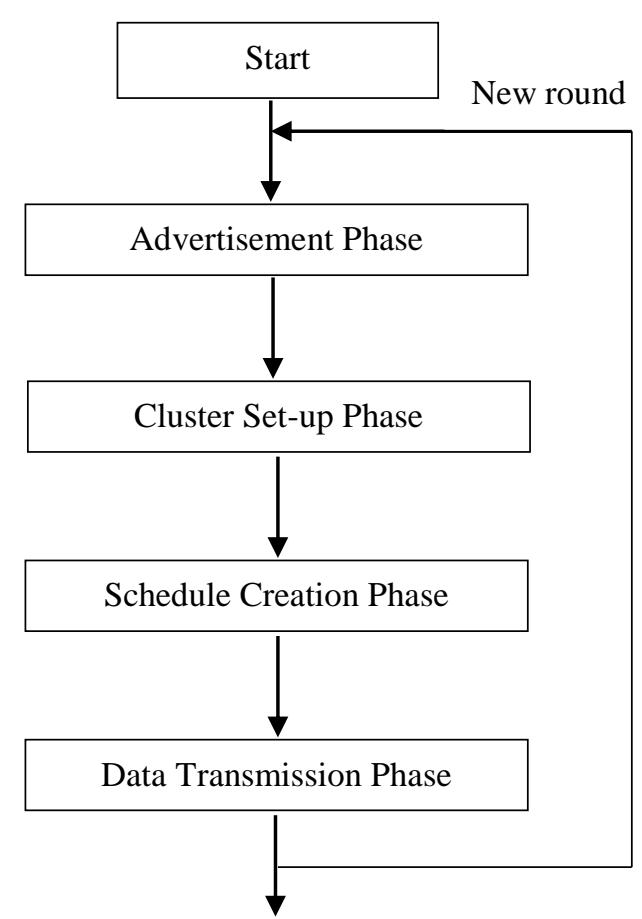

Data to the Base Station

Figure 3. LEACH algorithm flowchart [5]

\section{B. Node Scheduling Algorithm}

This algorithm [10] was proposed to minimize the active nodes and preserve the original sensing coverage. The process of this algorithm consists of two steps: selfscheduling step and sensing step. Self-scheduling step is composed of two stages. First, each node announces its location and hears to advertising messages from the other nodes to collect the position of each neighboring nodes. Second, each node computes its covering area and compares it with the covering area of its neighbors to decide if this node goes to sleep or not as shown in Fig. 4.

After each round, the remaining energy of the nodes is decreased with time and its sensing radius is gradually reduced. A threshold (W) of energy consumption is added for each sensor node as explained in [11].

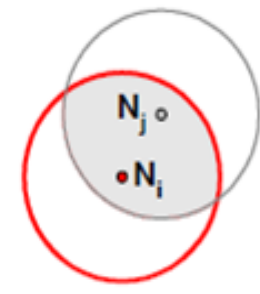

(a) $S(j) \cap S(i)$

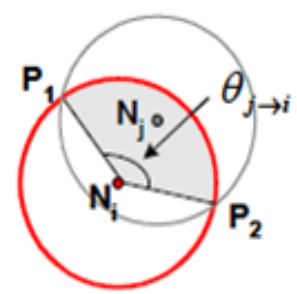

(b) $S_{j \rightarrow i}$ and $\theta_{j \rightarrow i}$
Figure 4. Illustration of the node coverage area [10] 


\section{CBCCP Protocol}

The prime goal of this protocol is to reduce energy in communication to prolong the network lifetime. CBCCP [8] begins its work by dividing the field into a static set of clusters arranged as levels. Each cluster selects one node randomly to act as $\mathrm{CH}$. In the first cluster (level 1), there is only one $\mathrm{CH}$, however, upper levels of clusters have one $\mathrm{CH}$ and some cluster coordinator nodes (CCOs). The number of CCOs in each level is equal to the number of cluster level -1 as shown in Fig. 5.

In this protocol, the data collected at each cluster are sent from $\mathrm{CH}$ to $\mathrm{CCO}$ in the upper level. This means that the $\mathrm{CH}$ of each cluster is responsible for collecting data of its level and $\mathrm{CCO}$ is responsible for receiving data of lower level. The method used for sending data reduces energy consumption and increases the network lifetime [8].

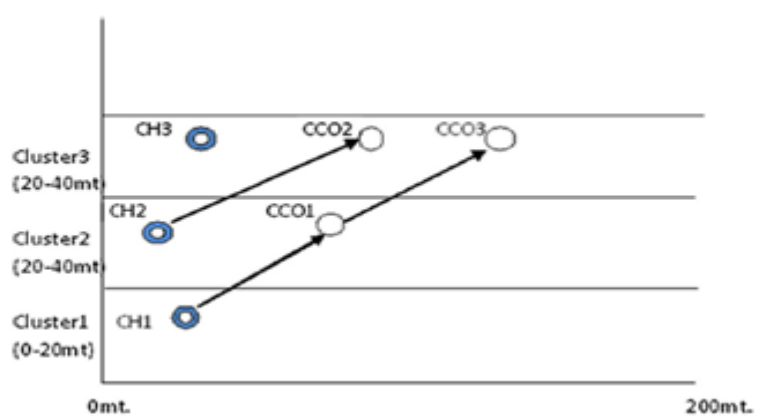

Figure 5. Inter-cluster communication with the help of cluster coordinators.

\section{MH-LEACH Algorithm}

The main goal of MH-LEACH [12] algorithm is to reduce the power consumption of the nodes and extend the network lifetime by establishment of multi-hop communication between clusters-head in a network. MH-LEACH finds possible routes for a cluster-head to transmit a data using other cluster-heads to reduce its power consumption. The possible routes for each cluster-head is indicated in its routing table as shown in Fig. 6. Received Signal Strength Indicator (RSSI) is used to establish the routing table of each cluster-head [12].

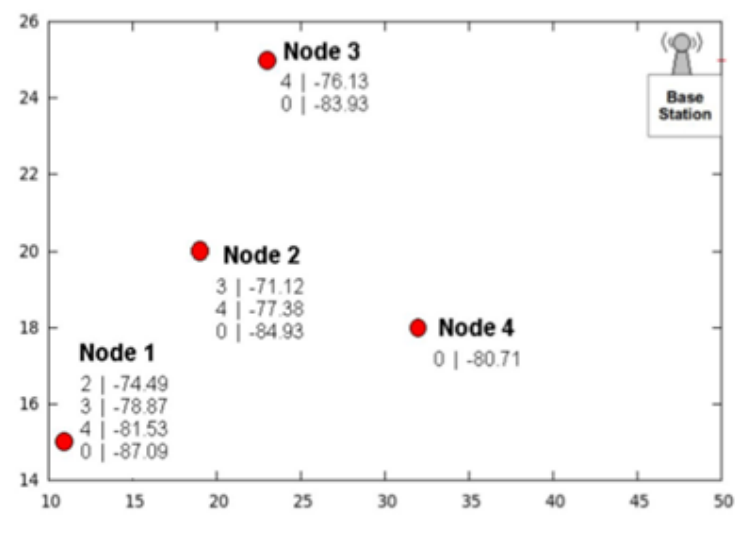

Figure 6. The routing table of each node.

\section{E. REECHD protocol}

The Rotating Energy Efficient Clustering for Heterogeneous Devices (REECHD) [13] protocol is proposed for heterogeneous WSNs, which are consisted of nodes with different transmission rates and different energy levels. The election of cluster head in REECHD not only takes into account the residual energy of nodes, but also the induced work of nodes. The induced work of nodes is estimated by using the node transmission rate. The node with lower transmission rate has a higher induced work. If a node with lower transmission rate and higher energy is selected as the cluster head, it will quickly deplete its energy. This will lead to quickly deplete the total amount of the cluster energy and decrease network lifetime [13].

\section{F. LEACH-AEC protocol}

As in LEACH [14], the operation of LEACH-AEC (LEACH with Adaptive Energy Consumption) is divided into rounds. Each round has two steps. The first is a setup step where nodes are divided into clusters, whereas the second step is a steady-state phase where data is collected and sent to the cluster head $(\mathrm{CH})$ and then to the base station. The selection of cluster head depends of the threshold T. the calculation of the threshold $\mathrm{T}$ is depending on the residual energy of nodes, the distance from sink, and the distances between cluster heads. LEACH-AEC uses also the multi-hop routing extension via A-Star algorithm to adaptive with routing in large WSN areas. This means that the cluster heads that located far from the sink, can communicate with the sink through other cluster heads, to decrease its energy consumption [14].

\section{THE PROPOSED PROTOCOL}

The main problem of WSNs is the network lifetime. To extend the network lifetime, we need an efficient routing and scheduling algorithm to enhance the energy used. In this paper, an energy-efficient chain-based cooperative sleep/wake-up routing protocol is proposed to prolong the network lifetime by using sleep/wake-up mechanism. The proposed protocol consists of two steps: scheduling step, which is performed using a sleep/wake-up mechanism, and a clustering step, which is based on cluster levels. The two steps are explained in detail next.

We suppose that the WSN consists of $\mathrm{N}$ sensor nodes randomly deployed in the sensing field as shown in Fig. 7. The sensor nodes are stationary; each node realizes its location and its neighbors and where they exist; each node is familiar with its coverage area. The coverage area of a node is known as the area that can be observed by a node, when this node is active. Each node belongs to exactly one cluster. After node-scheduling, each node can be off-duty (sleep) state or on-duty (wake-up) stated. During clustering, each node can be a normal node, a cluster head $(\mathrm{CH})$ or a cluster coordinator (CCO) [8].

\section{A. Scheduling Step}

As discussed above, the main goal of this step is to minimize the active nodes and preserve the original sensing coverage. In this step, each node announces its Position Advertise Message (PAM), which contains its id, position 
and sensing range to all neighbor nodes, and listens to the messages from its neighbors. The sleep/wake-up mechanism [10] works on the basis of comparing the sensing area of the node with its neighbors. If the sensor node is completely covered by its neighbors, this node goes to sleep without any problem in sensing data to reduce its energy consumption.

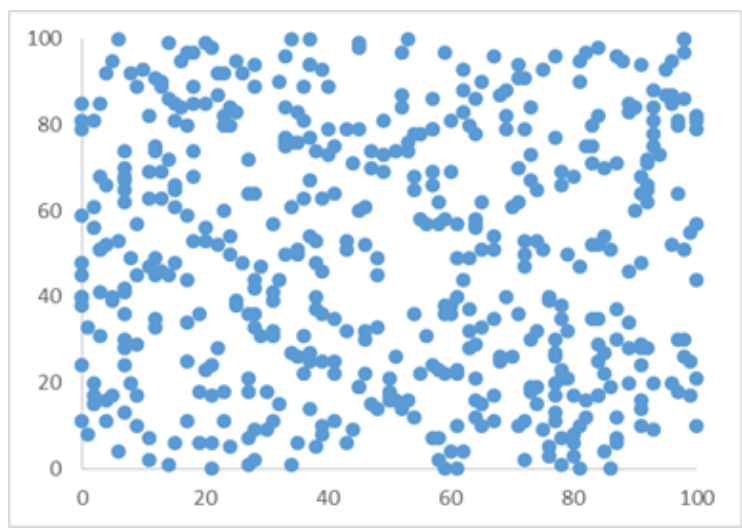

Figure 7. Sensor nodes deployed in the field.

The sleeping nodes can wake-up when the residual energy of the wake-up nodes is less than the proposed threshold energy by sending a wake-up command to the sleeping nearby nodes. The sleeping nodes wake-up and re-enter the self-scheduling phase to give a chance for the less energy nodes to sleep; at this point, another round starts as shown in Fig. 8.

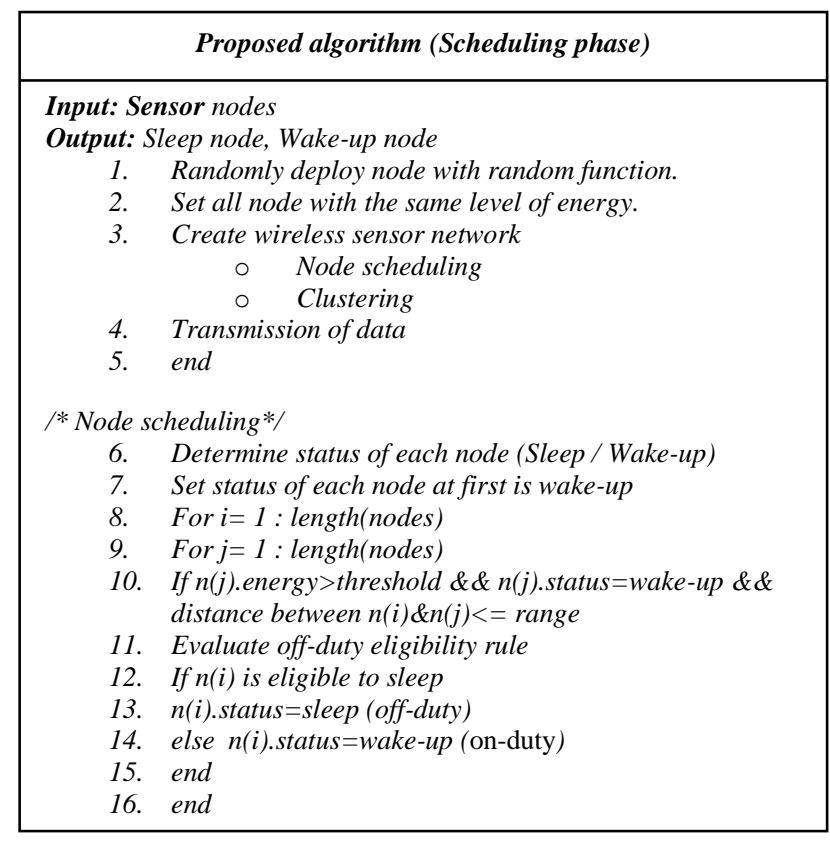

Figure 8. Scheduling phase algorithm.

\section{B. Clustering Phase}

The clustering phase starts once the network is installed to construct a static level of clusters. The clustering phase starts its work by dividing the field into sub areas (a static set of clusters) arranged as levels as shown in Fig. 9. For each cluster, one node is selected as a $\mathrm{CH}$ and a number of nodes is selected as a CCOs depending on the cluster level.

For example, suppose that the area of simulation is $200 \mathrm{~m}$ $* 200 \mathrm{~m}$. Then we can construct ten clusters, each with dimension of $200 \mathrm{~m} * 20 \mathrm{~m}$. In each cluster, one node is selected as a $\mathrm{CH}$ randomly. The first cluster has the dimension of $\left(0-200 \mathrm{~m}^{*} 0-20 \mathrm{~m}\right)$ and has one node acting as the $\mathrm{CH}$ to send data to the next cluster level (second cluster) with dimensions of $(0-200 \mathrm{~m} * 21-40 \mathrm{~m})$.

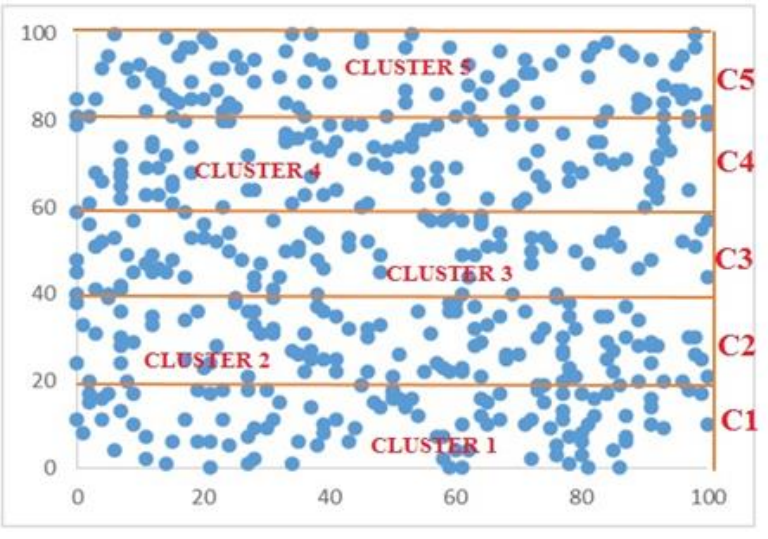

Figure 9. Cluster Creation.

The second cluster has one $\mathrm{CH}$ and one node acting as the $\mathrm{CCO}$ to receive data from the $\mathrm{CH}$ in the first cluster. This data is sent to the next $\mathrm{CCO}$ in the next cluster level (third cluster) with dimensions of $(0-200 m * 41-60 m)$. This data is continuously sent to the next $\mathrm{CCO}$ in the next cluster level until it is sent to the base station. The CCOs are the nodes, which used to send data of $\mathrm{CHs}$ to the base station. The numbers of CCOs in each cluster depend on the cluster level because each cluster must have one $\mathrm{CCO}$ for each cluster lying it to receive data. Therefore, if we have six clusters, the cluster number six must have five CCOs for each five cluster lying it to receive data from each cluster as shown in Fig. 5.

If the energy of any node is greater than a threshold level, this node can be selected as a $\mathrm{CH}$ or a $\mathrm{CCO}$. When the energy of any $\mathrm{CH}$ or any $\mathrm{CCO}$ is consumed and reached to the threshold level, a clustering process is affected and new $\mathrm{CHs}$ and CCOs are selected in all clusters. Then, the cluster is organized. After constructing the clusters, they communicate with each other to aggregate the data and send it to the base station. In each cluster, the local nodes perform the sensing task and transmit the data to the $\mathrm{CH}$ with the help of relay nodes. The $\mathrm{CHs}$ are responsible for aggregating the received data and transmitting it to the $\mathrm{CCO}$ in the next level cluster. The CCO send the received data to the next CCO in the upper cluster. The CCOs are the nodes, which used to send data of $\mathrm{CHs}$ to the base station. Finally, the data is sent to the base station through the CCOs [6]. The steps of the proposed protocol are shown in Fig. 10.

\section{PROTOCOL EVALUATION AND SIMULATION RESULTS}

The proposed protocol is implemented and evaluated using MATLAB R2013a. Our protocol is compared with the most two famous protocols: LEACH [3] and CBCCP [8]. The performance metrics used in our comparison are: the 
number of dead nodes, the number of sent packets to the base station, the sum of nodes energy and The number of alive nodes. The network specification used is shown in Table 1.

The number of dead nodes versus rounds is indicated in Fig. 11. From the figure, we can see that the proposed algorithm has the lowest number of dead nodes compared to the other two protocols. In the both LEACH and CBCCP protocols, nodes are dying fast as compared to the proposed protocol. The proposed protocol uses a sleep/wake-up mechanism which increases the node lifetime and thus decreases the number of dead nodes.

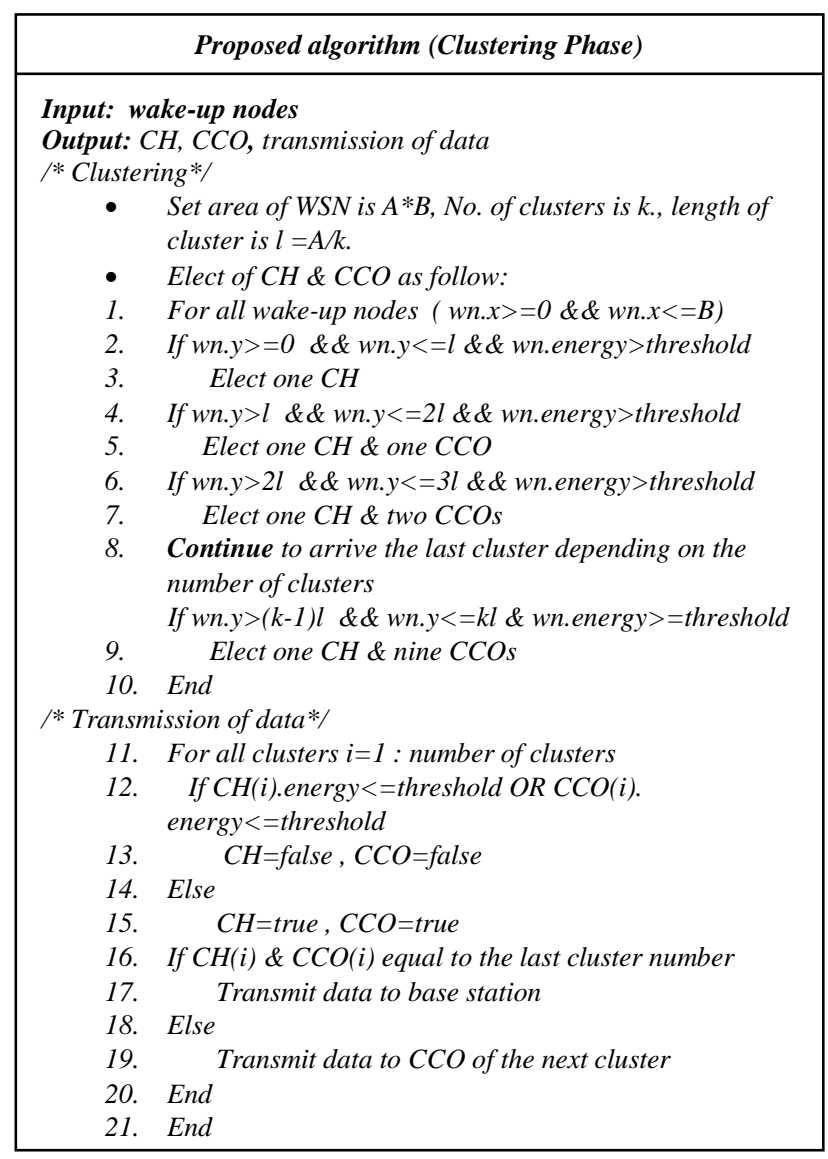

Figure 10. Clustering Phase algorithm.

TABLE 1

THE PARAMETERS AND ITS VALUES USED IN SIMULATION [6].

\begin{tabular}{|l|l|}
\hline \multicolumn{1}{|c|}{ Parameters } & Value \\
\hline Area & $200 \times 200 \mathrm{~m} 2$ \\
\hline Number of nodes & 1000 \\
\hline$\varepsilon f s$ & $10 \mathrm{pJ} / \mathrm{bit} / \mathrm{m} 2$ \\
\hline cmp & $0.0013 \mathrm{pJ} / \mathrm{bit} / \mathrm{m} 4$ \\
\hline 1 (length of data) & $4000 \mathrm{bit}$ \\
\hline Ee (Initial energy of the nodes) & $0.5 \mathrm{~J}$ \\
\hline ET (Transmitting energy) & $50 \mathrm{~nJ} / \mathrm{bit}$ \\
\hline ER (Reception Energy) & $50 \mathrm{~nJ} / \mathrm{bit}$ \\
\hline Ebf (Energy consumption in Beam Forming) & $5 \mathrm{~nJ} / \mathrm{bit}$ \\
\hline Sensing range, $\mathrm{r}$ & $10 \mathrm{~m}$ \\
\hline Threshold & $0.1 \mathrm{~J}$ \\
\hline
\end{tabular}

The sum of nodes energy versus rounds is shown in Fig. 12. The proposed algorithm outperforms the other two algorithms in the sum of nodes energy, which causes a prolonged network lifetime. This results from the use of a sleep/wake-up mechanism, which increases the nodes energy and nodes lifetime. On the contrary, in case of the other protocols, nodes are dying fast.

In Fig. 13, the numbers of sending packets to the base station versus rounds are shown. As the results show, LEACH has the highest number of sending packets to the base station because it uses more clusters than the proposed algorithm, which has a finite number of clusters (In the proposed protocol, the number of clusters is static and it is determined depending on the sensing area. In LEACH protocol, the number of cluster is determined randomly depending on the probability function). Because LEACH uses more clusters, it has many redundant packets. However, the proposed algorithm has a higher number of sending packets than CBCCP because the use of a sleep/wake-up mechanism that increases the network lifetime. Increasing network lifetime means that there are number of sensing nodes still alive. These sensing nodes perform its work and send data to the base station.

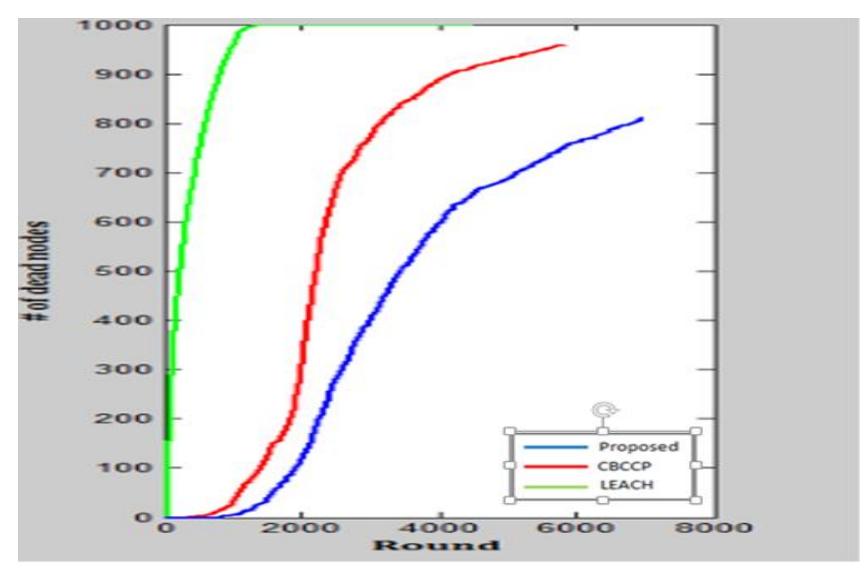

Figure 11. The number of dead node VS. round.

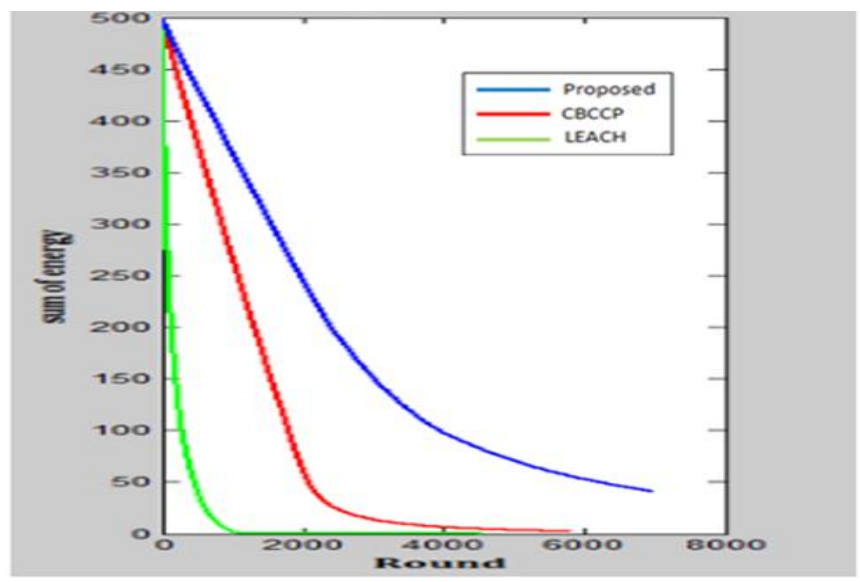

Figure 12. Sum of energy of node VS. round.

The number of alive nodes versus rounds is shown in Fig. 14. The nodes remain alive for long time in the proposed protocol, which causes a prolonged network lifetime. Our proposed protocol increases the nodes lifetime comparing both LEACH and CBCCP protocol. This results from the use 
of a sleep/wake-up mechanism, which increases the nodes lifetime. On the contrary, in case of the other protocols, nodes are dying fast.

In Fig. 15, the average energy consumption versus rounds is shown. This metric is important because the energy consumption of the network is proportional to the networks lifetime. The lower the energy consumption, the longer the networks lifetime. From this figure, it can be seen that our proposed algorithm has a lowest energy consumed comparing with LEACH and CBCCP. This because of the use of a sleep/wake-up mechanism, which decreases the nodes energy consumption and increases network lifetime.

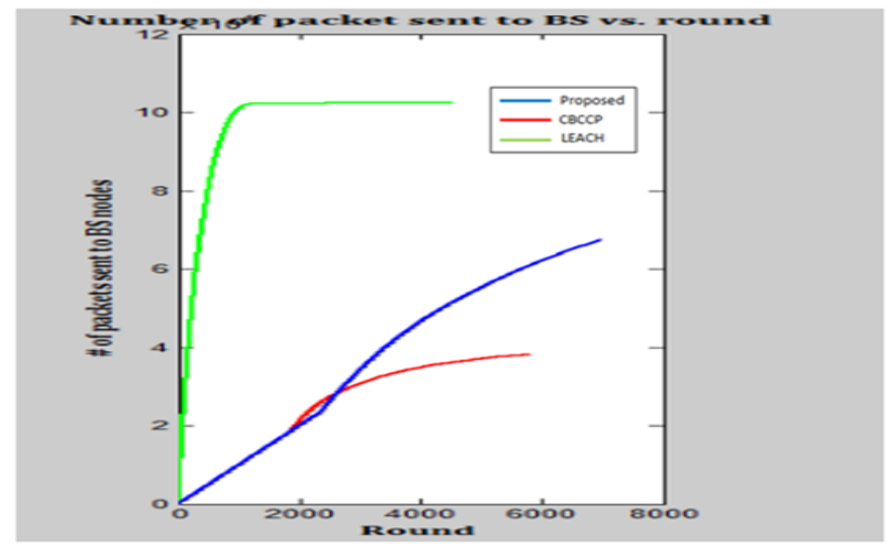

Figure 13. Number of packets sent to BS VS. round

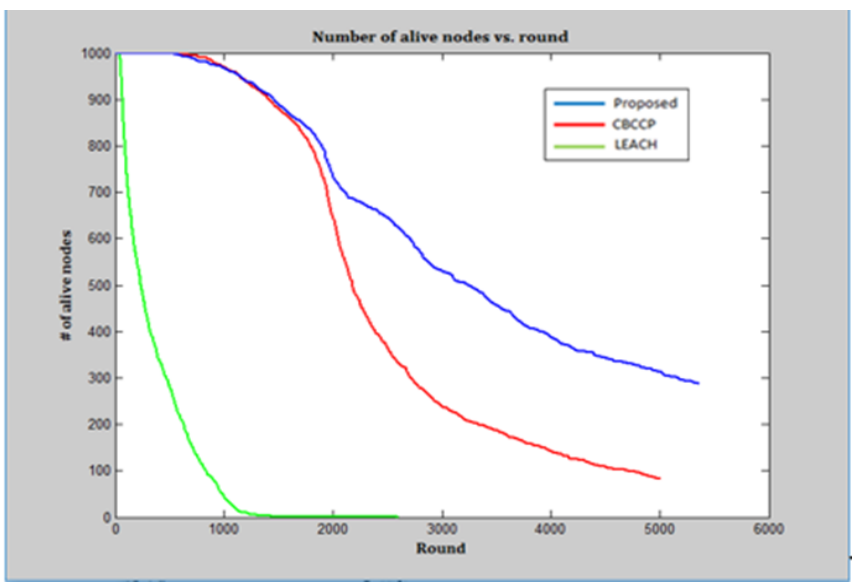

Figure 14. Number of alive nodes VS. round.

\section{CONCLUSION AND FUTURE WORKS}

In recent years, WSNs have a rapid development and they take a lot of research attention. The main essential parameter of WSNs is the network lifetime, so we need an energy efficient routing protocol to prolong the network lifetime. In this paper, energy efficient chain based cooperative sleep/wake-up routing protocol is proposed. This protocol reduces energy consumption and therefore increases the network lifetime. We compare this protocol with two efficient protocols, LEACH and CBCCP using MATLAB. The simulation results show that the proposed protocol exceeds the others in the number of dead nodes and the sum of nodes energy performance metrics. Our protocol assumed that the coverage area of each node is a circle. Practically, there are many factors can change the shape of the coverage area to asymmetrical shape such as weather and obstacles. Considering these factors is our future work.

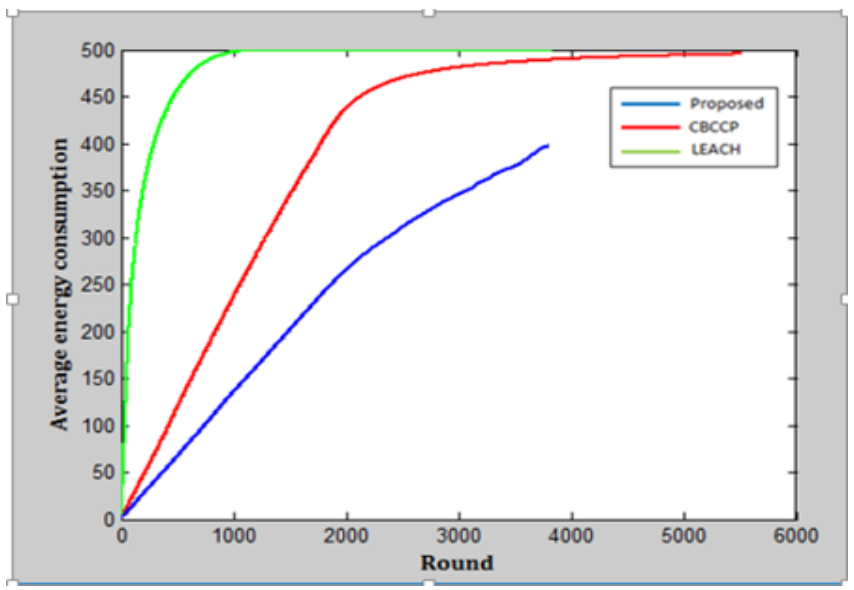

Figure 15. Average energy consumption VS. round.

\section{REFERENCES}

[1] J.N. Al-Karaki, A.E. Kamal, "Routing techniques in wireless sensor networks: a survey", IEEE Wirel. Commun., vol. 11, no. 6, pp. 6-28, 2004.

[2] F. Ye et al., "A Scalable Solution to Minimum Cost Forwarding in Large Sensor Networks," Proc. 10th Int'l. Conf. Comp. Commun. and Networks, 2001, pp. 304-09.

[3] W. Heinzelman, A. Chandrakasan, H. Balakrishnan, Energy-efficient communi-cation protocol for wireless microsensor networks, in: Proceedings of the 33rdHawaii International Conference on System Sciences, 4-7 January, 2000.

[4] Y. Xu, J. Heidemann, and D. Estrin, "Geography informed Energy Conservation for Ad-hoc Routing," Proc. 7th Annual ACM/IEEE Int'1. Conf. Mobile Comp. and Net., 2001, pp. 70-84.

[5] S. Lindsey and C. Raghavendra, "PEGASIS: Power-Efficient Gathering in Sensor Information Systems," IEEE Aerospace Conf. Proc., 2002, vol. 3, 9-16, pp. 1125-30.

[6] Fan Xiangning; Song Yulin, "Improvement on LEACH Protocol of Wireless Sensor Network," International Conference on Sensor Technologies and Applications, 2007, pp.260-264, 14-20 Oct. 2007.

[7] Ahmed Al-Baz and Ayman El-SAYED, "A new algorithm for cluster head selection in LEACH protocol for wireless sensor networks", International Journal of Communication Systems (IJCS), Volume 31, Issue 1, January 2018, https://doi.org/10.1002/dac.3407

[8] S. Rania, J. Malhotrab, R.Talwarc "Energy efficient chain based cooperative routing protocol for WSN," Appl. Soft Comput., vol. 35, Oct. 2015, pp. 386-397.http://dx.doi.org/10.1016/j.asoc.2015.06.034

[9] I. F. Akyildiz, W. Su, Y. Sankarasubramaniam, E. Cayirci, "A survey on sensor networks", IEEE Communications Magazine, 2002, pp. $102-$ 114.

[10] D. Tian and N.D. Georganas, A coverage-preserving node scheduling scheme for large wireless sensor networks, in: ACM Int'l Workshop on Wireless Sensor Networks and Applications (WSNA) (2002).

[11] Li Hongsheng, Liu Sumin and Hu Bing, "Research on Node Sleep/Wake-up Mechanism in WSN Based on Energy Threshold Setting, " International Conference on Wireless Communications, Networking and Mobile Computing, WiCom'09, Sept. 24-26, 2009, pp. $1-4$.

[12] Jos'e Henrique Brand ao Neto, Antoniel da Silva Rego, Andr'e Ribeiro Cardoso and Joaquim Celestino Jr, "MH-LEACH: A Distributed Algorithm for Multi-Hop Communication", The Thirteenth International Conference on Networks, 2014.

[13] M. Micheletti, L. Mostarda, and A. Piermarteri, "Rotatingenergyefficient clustering for heterogeneous devices (REECHD)," in Proc. 32nd IEEE Int. Conf. Adv. Inf. Netw. Appl. (AINA), Krakow, Poland, May 2018, pp. 213-220.

[14] M. Bajelan, H. Bakhshi, "An adaptive LEACH-based clustering algorithm for wireless sensor networks," Journal of communication engineering, Vol. 2, No. 4, Autumn 2013. 